\title{
Acúmulo de Nutrientes em Soja Convencional e Soja RR em Diferentes Tipos de Controle de Planta Daninha ${ }^{1}$
}

\author{
Nutrient Accumulation in Conventional and Glyphosate-Resistant Soybean under Different Types \\ of Weed Control
}

\author{
ZOBIOLE, L.H.S. ${ }^{2}$, OLIVEIRA JR., R.S. ${ }^{3}$, CONSTANTIN, J. ${ }^{3}$, OLIVEIRA JR, A. ${ }^{4}$, CASTRO, C. ${ }^{4}$, \\ OLIVEIRA, F.A. ${ }^{4}$, KREMER, R.J. ${ }^{5}$, MOREIRA, A. ${ }^{4}$ e ROMAGNOLI, L.M. ${ }^{6}$
}

\begin{abstract}
RESUMO - O cultivo da soja - Glycine max (Roundup Ready ${ }^{\oplus}$ - RR) tem aumentado, e pouco tem sido relatada a influência do glyphosate sobre o estado nutricional das plantas. O objetivo deste trabalho foi comparar o acúmulo de nutrientes em diferentes estádios fenológicos entre os cultivares BRS 184 (convencional) e BRS 243 RR (transgênico), com mesmo ciclo vegetativo e reprodutivo, sob diferentes sistemas de manejo de plantas daninhas (capina e herbicida). O acúmulo de nutrientes e matéria seca na soja convencional foi superior ao da soja RR tratada com glyphosate, indicando que um nível maior de nutrientes pode ser requerido pelos cultivares RR para atingir a eficiência fisiológica e, provavelmente, uma nova recomendação de adubação para as culturas RR deve ser levada em consideração devido à redução da eficiência nutricional imposta pelo glyphosate.
\end{abstract}

Palavras-chave: glyphosate, absorção de nutrientes, Glycine max.

\begin{abstract}
The cultivation of soybean-Glycine max (Roundup Ready ${ }^{\circledR}-R R$ ) has increased and little has been reported on the influence of glyphosate on the nutritional status of the plants. The aim of this work was to compare nutrient accumulation at different phenological stages between the cultivars BRS 184 (conventional) and BRS 243 RR (transgenic), with the same crop cycle, under different weed management systems (hand weed and herbicide). Nutrient accumulation and dry matter in conventional soybean was superior to that in the glyphosate-treated $R R$ soybean, indicating that a higher level of nutrients might be required for the RR cultivars to achieve physiological efficiency and a new fertilizer recommendation for RR crops may be considered, due to the reduced nutritional efficiency imposed by glyphosate.
\end{abstract}

Keywords: glyphosate, nutrient uptake, Glycine max.

\section{INTRODUÇÃO}

A produção de soja resistente ao glyphosate (RR) continua a crescer anualmente no Brasil e no mundo, principalmente pelos beneficios dessa tecnologia no manejo de plantas daninhas. Atualmente, a área plantada de soja RR no Brasil ultrapassa os dez milhões de hectares. Embora essa tecnologia seja usada em muitos países, as recomendações de adubação ainda são incipientes. Trabalhos realizados por Zobiole et al. (2010a) demonstram que o estado nutricional da soja RR pode ser influenciado pelo glyphosate, com significativa diminuição nos teores foliares de macro e micronutrientes. O efeito dessa diminuição

\footnotetext{
Recebido para publicação em 28.11.2010 e aprovado em 31.6.2011.

2 Doutorando do curso do Programa de Pós-Graduação em Agronomia, Universidade Estadual de Maringá - UEM, Av. Colombo, 5790, Bloco J45, 87020-900, Maringá, PR, <lhzobiole@uol.com.br >; ${ }^{3}$ Professor Associado do Dep. de Agronomia, UEM; ${ }^{4}$ Embrapa Soja, Caixa Postal 231, 86001-970 Londrina-PR, ${ }^{5}$ United States Department of Agriculture, Agricultural Research Service, Cropping Systems \& Water Quality Research Unit, Columbia, MO 65211, USA; ${ }^{6}$ Eng--Agr ${ }^{\circ}$., Universidade Estadual de Londrina - UEL, Londrina-PR.
} 
poderia ser atribuído à redução das reações fotossintéticas (Kitchen et al., 1981; Reddy et al., 2004) ou à imobilização de micronutrientes catiônicos ( $\mathrm{Cu}, \mathrm{Fe}, \mathrm{Mn}$ e $\mathrm{Zn}$ ) pelo glyphosate, devido à habilidade desse herbicida em formar complexos insolúveis entre glyphosate-metal (Jaworski, 1972; Coutinho \& Mazo, 2005). Bott et al. (2008) também observaram reduções significativas do teor foliar de Mn e da biomassa de raiz após a aplicação de glyphosate na soja RR. Embora os efeitos do glyphosate sobre a soja RR sejam dependentes de fatores como variedade, grupo de maturação, época de aplicação e dose, trabalhos anteriores desenvolvidos por nosso grupo de trabalho já demonstraram o efeito deletério do glyphosate sobre a nodulação de um grande número de variedades de soja RR cultivadas no Brasil (Dvoranen et al., 2008; Oliveira Jr. et al., 2008; Zobiole et al. 2010b).

Um estudo subsequente, visando avaliar a influência do aumento da dose de glyphosate na soja RR, revelou que o aumento da dose de glyphosate promoveu redução na absorção de água (Zobiole et al., 2010c), ocasionando reduções na absorção e distribuição de nutrientes. Para Reddy et al. (2004), as injúrias causadas na soja RR podem ser relacionadas ao acúmulo de AMPA (ácido aminometilfosfônico) - primeiro metabólito fitotóxico do glyphosate.

Considerando o fato de que agricultores relatam que cultivares de soja RR são visualmente injuriados pelo glyphosate (Zablotowicz $\&$ Reddy, 2007) e que existem poucos resultados sobre a interferência do glyphosate na nutrição mineral da soja RR, o objetivo deste trabalho foi avaliar o acúmulo de macro $(\mathrm{N}, \mathrm{P}$, $\mathrm{K}, \mathrm{Ca}, \mathrm{Mg}$ e $\mathrm{S}$ ) e micronutrientes $(\mathrm{B}, \mathrm{Cu}, \mathrm{Fe}$, $\mathrm{Mn}$ e $\mathrm{Zn}$ ) em um cultivar convencional submetido à aplicação de fluazifop-p-butyl+ fomesafen na soja convencional e de glyphosate na soja RR submetida, em relação às mesmas variedades conduzidas sob capina manual.

\section{MATERIAL E MÉTODOS}

O experimento foi realizado durante o ano agrícola 2007/2008, na área experimental da Embrapa Soja, Londrina-PR, situada na latitude de $23^{\circ} 23^{\prime} \mathrm{S}$ e longitude de $51^{\circ} 11^{\prime} \mathrm{W}$.
A temperatura e pluviosidade média foram de $22,7^{\circ} \mathrm{C}$ e $181 \mathrm{~mm}$, respectivamente. O solo da área experimental foi classificado como Latossolo Vermelho distroférrico (Embrapa, 2006), com $766 \mathrm{~g} \mathrm{~kg}^{-1}$ de argila, $182 \mathrm{~g} \mathrm{~kg}^{1}$ de silte, $52 \mathrm{~g} \mathrm{~kg}^{-1}$ de areia e as seguintes características químicas da camada arável (0-20 cm): $\mathrm{pH} \mathrm{CaCl}$ de 5,4; 16,6 $\mathrm{mg} \mathrm{dm}^{-3}$ de $\mathrm{P}$ (Mehlich-1); 0,7 $\mathrm{cmol}_{\mathrm{c}} \mathrm{dm}^{-3} \mathrm{de} \mathrm{K} ; 4,4 \mathrm{cmol}_{\mathrm{c}} \mathrm{dm}^{-3}$ de Ca; 2,8 $\mathrm{cmol}_{\mathrm{c}} \mathrm{dm}^{-3}$ de $\mathrm{Mg} ; 3,8 \mathrm{cmol}_{\mathrm{c}} \mathrm{dm}^{-3} \mathrm{de}$ $\mathrm{H}+\mathrm{Al}$; e $11,0 \mathrm{~g} \mathrm{dm}^{-3}$ de $\mathrm{C}$ orgânico. A caracterização química foi feita de acordo com os procedimentos propostos em Embrapa (1999).

A soja (BRS 184 e BRS 243 RR) foi implantada em semeadura direta com densidade inicial de 18 sementes por metro linear, 10 dias após a dessecação com glyphosate [(480 g L-1 equivalente ativo (e.a)]. A adubação de semeadura foi constituída de $40 \mathrm{~kg} \mathrm{ha}^{-1}$ de $\mathrm{K}_{2} \mathrm{O}$ e $40 \mathrm{~kg} \mathrm{ha}^{-1}$ de $\mathrm{P}_{2} \mathrm{O}_{5}$. Antes da semeadura, as sementes de soja foram tratadas com $100 \mathrm{~mL}$ para $100 \mathrm{~kg}^{-1}$ de sementes com a mistura de $13,5 \mathrm{~g} \mathrm{~L}^{-1}$ de cobalto e $135 \mathrm{~g} \mathrm{~L}^{-1} \mathrm{de}$ molibdênio. Fez-se também a inoculação com $300 \mathrm{~mL}$ para $100 \mathrm{~kg}^{-1}$ de sementes da cultura de Bradyrhizobium elkanii, estirpes SEMIA 587 e SEMIA 5019, na concentração de $5 \times 10^{9}$ rizóbios por grama.

Quando as plântulas atingiram o estádio V1, foi realizado o desbaste, deixando-se, em média, 15 plantas por metro linear, com densidade populacional de aproximadamente 334.000 plantas ha ${ }^{-1}$. Os demais tratos culturais foram realizados segundo as recomendações descritas em Embrapa (2007).

O experimento foi delineado em blocos ao acaso com cinco repetições, utilizando um esquema fatorial ( $2 \times 2)$, sendo o primeiro fator representado pelos cultivares (BRS 184 e BRS 243 RR) e o segundo pelo manejo de plantas daninhas (herbicida ou capina manual). Independentemente dos tratamentos aplicados, todas as parcelas foram manualmente capinadas a cada sete dias, para que não ocorresse matocompetição.

Os herbicidas foram aplicados aos 24 dias após a emergência (DAE), no estádio V4, estando as plantas daninhas de folhas largas no estádio predominante de duas folhas 
verdadeiras. Para a soja convencional (BRS 184), foram utilizados os herbicidas fluazifop-p-butyl (200 $\left.\mathrm{g} \mathrm{L}^{-1}\right)+$ fomesafen (240 $\left.\mathrm{g} \mathrm{L}^{-1}\right)$, em mistura de tanque. Quanto à soja transgênica (BRS $243 \mathrm{RR}$ ), foi aplicado o herbicida glyphosate, na dose de $720 \mathrm{~g}^{\mathrm{ha}^{-1}}$ de e.a. As aplicações dos herbicidas foram realizadas com um pulverizador costal de pressão constante à base de $\mathrm{CO}_{2}$, equipado com pontas tipo leque XR-110.02, pressão de $2,0 \mathrm{kgf} \mathrm{cm}{ }^{-2}$, com volume de calda equivalente a $190 \mathrm{~L} \mathrm{ha}^{-1}$. As condições climáticas no momento da aplicação eram de temperatura entre 24 e $28{ }^{\circ} \mathrm{C}$, umidade relativa do ar entre 80 e $90 \%$, solo úmido e velocidade do vento entre 6 e $8 \mathrm{~km} \mathrm{~h}^{-1}$.

As unidades experimentais foram constituídas de parcelas com 12 linhas de semeadura, espaçadas de 0,45 m entre fileiras, com área de $90 \mathrm{~m}^{2}(6 \times 15 \mathrm{~m})$, tomando-se como área útil as quatro linhas centrais, exceto $1,5 \mathrm{~m}$ de cada extremidade. Em cada tratamento, foram coletadas 12 plantas em diferentes estádios fenológicos: V2, V4, V6, R1, R3, R4, R5.1, R5.3, R5.5, R6, R7, R8 (17, 24, 31, 53, 60, 67, 78, 90, 97, 107, 113 e $123 \mathrm{DAE}$, respectivamente - a partir do estádio V6, as plantas entraram em floração). As coletas foram realizadas ao acaso, em seis plantas, numa área de $1,0 \mathrm{~m}^{2}$ na área útil da parcela, procedendo-se à separação em folhas, pecíolos, caules e, quando existentes, vagens e grãos. A área foliar foi determinada em dois estádios: a) início do crescimento vegetativo - V2 e final do florescimento e início da formação de vagens - R3, utilizando-se integrador de área foliar, modelo LI-3100. Após cada coleta, as diversas partes das plantas foram lavadas com água deionizada, secas em estufa com circulação forçada de ar a $65^{\circ} \mathrm{C}$ e, posteriormente, moídas em moinho tipo Wiley. As análises químicas dos macro e micronutrientes nas amostras foram realizadas segundo metodologia descrita por Sarruge \& Haag (1974).

A colheita foi realizada aos $125 \mathrm{DAE}$, com uma colhedora de parcelas Wintersteiger, modelo Classic, em uma área útil da parcela de $16,2 \mathrm{~m}^{2}$, constituída das três linhas centrais, descontando as extremidades. A produtividade foi calculada em $\mathrm{kg} \mathrm{ha}^{-1}$ a $13 \%$ de umidade.
Os dados foram submetidos à análise de variância (ANOVA); quando os valores de $\mathrm{F}$ foram significativos $(\mathrm{P}<0.01)$, análises de regressão foram realizadas. O ajuste para acúmulo de matéria seca total, peso de grãos, da área foliar e dos nutrientes em função do tempo foi realizado por meio do modelo de Gaussian com três variáveis, descrito a partir da seguinte equação genérica:

$$
\hat{y}=a e^{\left[-0,5\left(\frac{x-x_{0}}{b}\right)^{2}\right]}
$$

em que: $\hat{y}$ = acúmulo de variável analisada; $\mathrm{a}=$ valor de máximo acúmulo; $\mathrm{x}_{0}=$ valor de $\mathrm{x}$, em DAE, que proporciona o máximo em ; e b = amplitude no valor de $\mathrm{x}$, em DAE, entre $\mathrm{o}$ ponto de inflexão e o ponto de máximo.

Entretanto, a partir do modelo ajustado foi possivel determinar o valor do ponto de inflexão (PI) na curva, da seguinte forma:

$$
\mathrm{PI}=\mathrm{x}_{0}-\mathrm{b}
$$

Matematicamente, o PI corresponde ao valor de $\mathrm{x}$ em que a curvatura do modelo ajustado muda de sinal; na prática, isso corresponde ao valor de $\mathrm{x}$, em DAE, em que a taxa de acúmulo diário, ainda que positiva, passa a decrescer.

\section{RESULTADOS E DISCUSSÃO}

Independentemente do cultivar, o incremento de matéria seca (MS) foi lento até o estádio R1/R3, que ocorreu, aproximadamente, aos $31 \mathrm{DAE}$ (Figura 1). Após esse estádio, houve maior incremento de MS; a partir dele, a produção de MS atingiu valores máximos entre 112 e $116 \mathrm{DAE}$, com 24,96,24,42,24,06 e 20,59 g por planta de MS total para os tratamentos T1, T2, T3 e T4, respectivamente (Tabela 1). Resultados semelhantes foram encontrados por Pedrinho Júnior et al. (2004), com acúmulo máximo aos 104 DAE, com 36,6 g por planta.

O BRS 243 RR, na presença do herbicida glyphosate (T4), acumulou menos MS e área foliar que os demais tratamentos (Figuras $1 \mathrm{e}$ 2; Tabelas 2 e 3). Na comparação entre os 
Tabela 1 - Estimativas dos parâmetros dos modelos ajustados para o acúmulo de matéria seca total e de grãos em função do tempo e os respectivos valores do ponto de inflexão (PI)

\begin{tabular}{|c|c|c|c|c|c|}
\hline \multirow{3}{*}{ Variáveis dependentes } & \multicolumn{3}{|c|}{ Estimativa dos parâmetros do modelo ajustado ${ }^{3 /}$} & \multirow{2}{*}{$\begin{array}{c}\text { PI } \\
\left(\mathrm{X}_{0}-\mathrm{b}\right)\end{array}$} & \multirow{3}{*}{$\mathrm{R}^{2}$} \\
\hline & a & $\mathrm{X}_{0}$ & $\mathrm{~B}$ & & \\
\hline & (g por planta) & \multicolumn{3}{|c|}{ (dias após a emergência) } & \\
\hline \multicolumn{6}{|l|}{ MS total ${ }^{1 /}$} \\
\hline $\mathrm{T} 1$ & 24,96 & 115,0 & 35,5 & 79 & $0,99 *$ \\
\hline $\mathrm{T} 2$ & 24,42 & 111,9 & 34,2 & 77 & $0,99 *$ \\
\hline T3 & 24,06 & 116,0 & 35,9 & 80 & $0,99 *$ \\
\hline $\mathrm{T} 4$ & 20,59 & 114,9 & 34,9 & 80 & $0,99 *$ \\
\hline \multicolumn{6}{|l|}{ MS grãos ${ }^{2 / 1}$} \\
\hline $\mathrm{T} 1$ & 12,89 & 122,6 & 12,7 & 109 & $0,99 *$ \\
\hline $\mathrm{T} 2$ & 12,61 & 122,5 & 13,7 & 108 & $0,99^{*}$ \\
\hline $\mathrm{T} 3$ & 10,55 & 122,4 & 12,0 & 110 & $0,99^{*}$ \\
\hline $\mathrm{T} 4$ & 8,17 & 122,5 & 12,4 & 110 & $0,99^{*}$ \\
\hline
\end{tabular}

1' Soma da MS das folhas, dos pecíolos, do caule, das vagens e dos grãos. ${ }^{2}$ MS somente dos grãos. ${ }^{3 /} \mathrm{a}=$ corresponde ao valor de máximo acúmulo; $\mathrm{x}_{0}=$ corresponde ao valor de $\mathrm{x}$, em DAE, que proporciona o máximo em $\quad$; b = corresponde à amplitude no valor de $\mathrm{x}$, em $\mathrm{DAE}$, entre o ponto de inflexão e o ponto de máximo; T1 = BRS 184 + capina manual; T2 = BRS 184 + herbicida seletivo; T3 = BRS 243 RR +

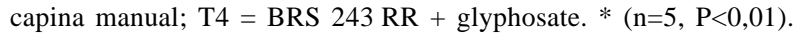

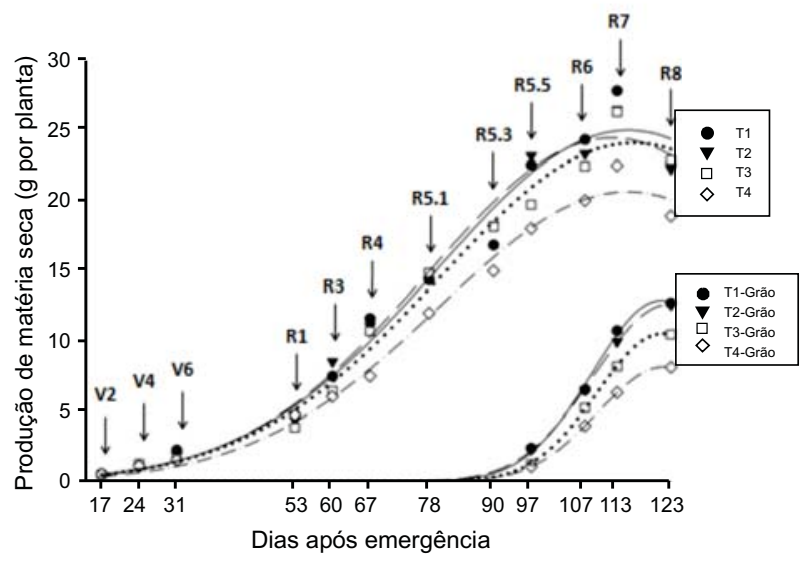

Figura 1 - Acúmulo de matéria seca total e de grãos de soja em função do tempo e de diferentes tratamentos. T1 = BRS 184 + capina manual (linha sólida); T2 = BRS 184 + herbicida seletivo (linha pontilhada); T3 = BRS $243 \mathrm{RR}+$ capina manual (linha tracejada); T4 = BRS 243 RR + glyphosate (linha ponto-tracejada).

cultivares, a área foliar do BRS 184 foi superior à do transgênico (Tabela 2). Esses resultados são confirmados por outros autores, que também encontraram reduções na biomassa seca da parte aérea e de raízes da soja $R R$ com o uso de glyphosate nas doses de $1.200 \mathrm{~g} \mathrm{ha}^{-1}$ (Zobiole et al., 2010a), $1.680 \mathrm{~g} \mathrm{ha}^{-1}$ (Reddy et al., 2000) e $6.300 \mathrm{~g} \mathrm{ha}^{-1}$ de e.a. (King et al., 2001).
O menor acúmulo de biomassa causada pelo glyphosate na soja RR pode ser atribuído ao acúmulo do ácido aminometilfosfônico (AMPA), seu principal metabólito (Reddy et al., 2004), ou às reduções na taxa fotossintética (Zobiole et al., 2010c) e na biodisponibilidade de nutrientes nos tecidos foliares (Cakmak et al., 2009; Zobiole et al., 2010a). Esses resultados

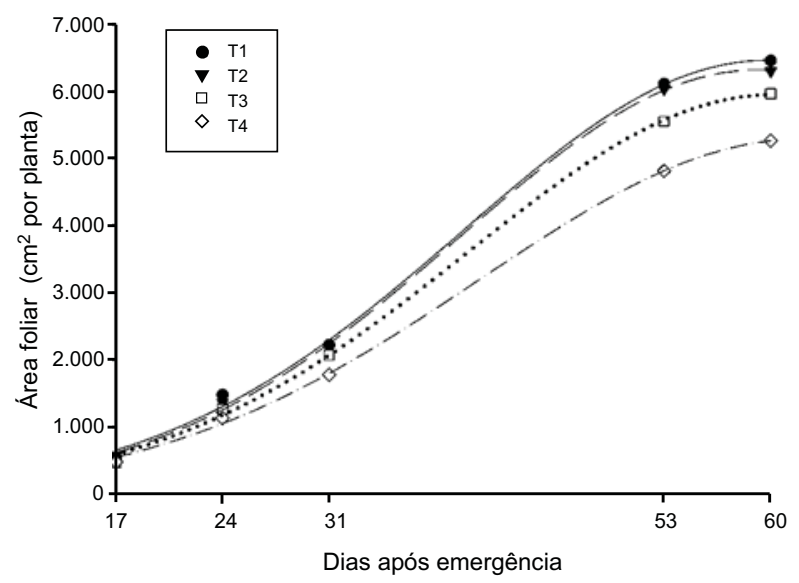

Figura 2 - Área foliar da soja em função do tempo e de diferentes tratamentos. T1 = BRS 184 + capina manual (linha sólida); T2 = BRS 184 + herbicida seletivo (linha pontilhada); T3 = BRS 243 RR + capina manual (linha tracejada); T4 = BRS 243 RR + glyphosate (linha ponto-tracejada). 
Tabela 2 - Estimativas dos parâmetros dos modelos ajustados para área foliar da soja em função do tempo e os respectivos valores do ponto de inflexão (PI)

\begin{tabular}{|c|c|c|c|c|c|}
\hline \multirow{3}{*}{ Tratamento } & \multicolumn{3}{|c|}{ Estimativa dos parâmetros do modelo ajustado ${ }^{\frac{1 /}{}}$} & \multirow{2}{*}{$\begin{array}{c}\text { PI } \\
\left(\mathrm{X}_{0}-\mathrm{b}\right)\end{array}$} & \multirow{3}{*}{$\mathrm{R}^{2}$} \\
\hline & $\mathrm{a}$ & $\mathrm{X}_{0}$ & $\mathrm{~B}$ & & \\
\hline & $\left(\mathrm{cm}^{2}\right.$ por planta $)$ & \multicolumn{3}{|c|}{ (dias após a emergência) } & \\
\hline $\mathrm{T} 1$ & $6.461,67$ & 59,75 & 19,98 & 39 & $0,99^{*}$ \\
\hline $\mathrm{T} 2$ & $6.318,55$ & 59,10 & 19,54 & 39 & $0,99 *$ \\
\hline $\mathrm{T} 3$ & $5.950,83$ & 60,29 & 20,14 & 40 & $0,99 *$ \\
\hline T4 & $5.292,31$ & 62,22 & 21,25 & 40 & $0,99^{*}$ \\
\hline
\end{tabular}

${ }^{1 /} \mathrm{a}=$ corresponde ao valor de máximo acúmulo; $\mathrm{x}_{0}=$ corresponde ao valor de $\mathrm{x}$, em DAE, que proporciona o máximo em $; \mathrm{b}=$ corresponde à amplitude no valor de $\mathrm{x}$, em DAE, entre o ponto de inflexão e o ponto de máximo; T1 = BRS $184+$ capina manual; T2 = BRS $184+$ herbicida seletivo; T3 = BRS $243 \mathrm{RR}+$ capina manual; T4 = BRS $243 \mathrm{RR}+$ glyphosate. ${ }^{*}(\mathrm{n}=5, \mathrm{P}<0.01)$.

Tabela 3 - Estimativas dos parâmetros dos modelos ajustados para o acúmulo de macronutrientes primários em função do tempo e os respectivos valores do ponto de inflexão (PI)

\begin{tabular}{|c|c|c|c|c|c|}
\hline \multirow{3}{*}{ Tratamento $^{\underline{1}}$} & \multicolumn{3}{|c|}{ Estimativa dos parâmetros do modelo ajustado ${ }^{\underline{2}}$} & \multirow{2}{*}{$\begin{array}{c}\text { PI } \\
\left(\mathrm{X}_{0}-\mathrm{b}\right)\end{array}$} & \multirow{3}{*}{$\mathrm{R}^{2}$} \\
\hline & $\mathrm{a}$ & $\mathrm{X}_{0}$ & $\mathrm{~B}$ & & \\
\hline & (mg por planta) & \multicolumn{3}{|c|}{ (dias após a emergência) } & \\
\hline \multicolumn{6}{|c|}{ Nitrogênio } \\
\hline $\mathrm{T} 1$ & 705,92 & 112,14 & 34,17 & 78 & $0,99^{*}$ \\
\hline $\mathrm{T} 2$ & 691,54 & 108,51 & 31,76 & 79 & $0,99 *$ \\
\hline T3 & 626,22 & 112,02 & 35,00 & 77 & $0,99^{*}$ \\
\hline $\mathrm{T} 4$ & 571,50 & 103,28 & 27,22 & 76 & $0,99 *$ \\
\hline \multicolumn{6}{|c|}{ Fósforo } \\
\hline $\mathrm{T} 1$ & 79,11 & 117,15 & 33,05 & 84 & $0,98^{*}$ \\
\hline $\mathrm{T} 2$ & 75,44 & 111,06 & 31,01 & 80 & $0,98^{*}$ \\
\hline T3 & 65,70 & 113,50 & 32,90 & 81 & $0,99 *$ \\
\hline $\mathrm{T} 4$ & 61,26 & 105,77 & 25,15 & 81 & $0,99^{*}$ \\
\hline \multicolumn{6}{|c|}{ Potássio } \\
\hline $\mathrm{T} 1$ & 385,93 & 107,13 & 36,47 & 71 & $0,99^{*}$ \\
\hline $\mathrm{T} 2$ & 351,65 & 101,78 & 34,35 & 67 & $0,99^{*}$ \\
\hline T3 & 369,56 & 109,02 & 36,47 & 72 & $0,98^{*}$ \\
\hline $\mathrm{T} 4$ & 328,79 & 104,20 & 31,56 & 73 & $0,99^{*}$ \\
\hline
\end{tabular}

1’ Somatório da MS das folhas, dos pecíolos, do caule, das vagens e dos grãos. 르a = corresponde ao valor de máximo acúmulo; $\mathrm{x}_{0}=$ corresponde ao valor de $\mathrm{x}$, em DAE, que proporciona o máximo em ; b = corresponde à amplitude no valor de $\mathrm{x}$, em DAE, entre o ponto de inflexão e o ponto de máximo; T1 = BRS 184 + capina manual; T2 = BRS 184 + herbicida seletivo; T3 = BRS 243 RR + capina manual; $\mathrm{T} 4=$ BRS $243 \mathrm{RR}+$ glyphosate. $*(\mathrm{n}=5, \mathrm{P}<0.01)$.

também encontram respaldo nos trabalhos de Bott et al. (2008), os quais observaram que a aplicação do glyphosate na soja RR reduziu significativamente a biomassa e a elongação da raiz.

A produção de grãos, por sua vez, teve início a partir dos $78 \mathrm{DAE}$ (estádio R5.1), com acúmulo máximo de 12,90, 12,61, 10,55 e $8,17 \mathrm{~g}$ por planta para os tatamentos $\mathrm{T} 1 \mathrm{a} \mathrm{T} 4$, respectivamente, observado entre 122 e
123 DAE (Figura 1; Tabela 1). Da mesma forma que o observado para o acúmulo de MS e área foliar, independentemente do tratamento aplicado, o cultivar BRS 184 submetido ao tratamento químico convencional acumulou mais MS nos grãos que o cultivar transgênico submetido ao glyphosate. No entanto, comparando apenas os tratamentos com o cultivar transgênico, observou-se que o uso de glyphosate levou à redução de $23 \%$ no acúmulo de MS dos grãos (Tabela 1). 
Zobiole et al. (2010d) relataram que o menor acúmulo de biomassa na soja RR na presença do glyphosate pode ser consequência do menor acúmulo e disponibilidade de nutrientes, causados pela propriedade
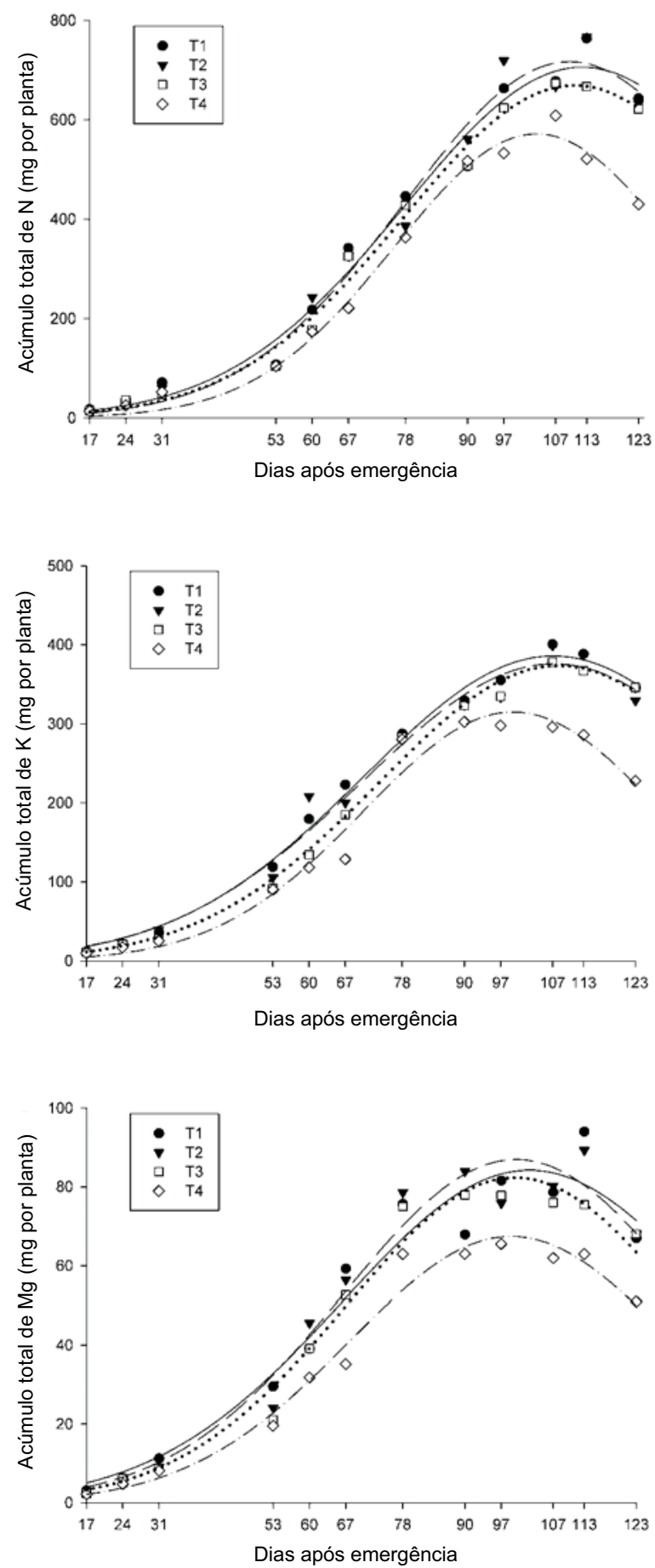

quelante desse herbicida. Diversos estudos têm mostrado que o glyphosate altera a disponibilidade dos micronutrientes nas plantas (Franzen et al., 2003; Bott et al., 2008; Johal \& Huber, 2009; Zobiole et al., 2010a).
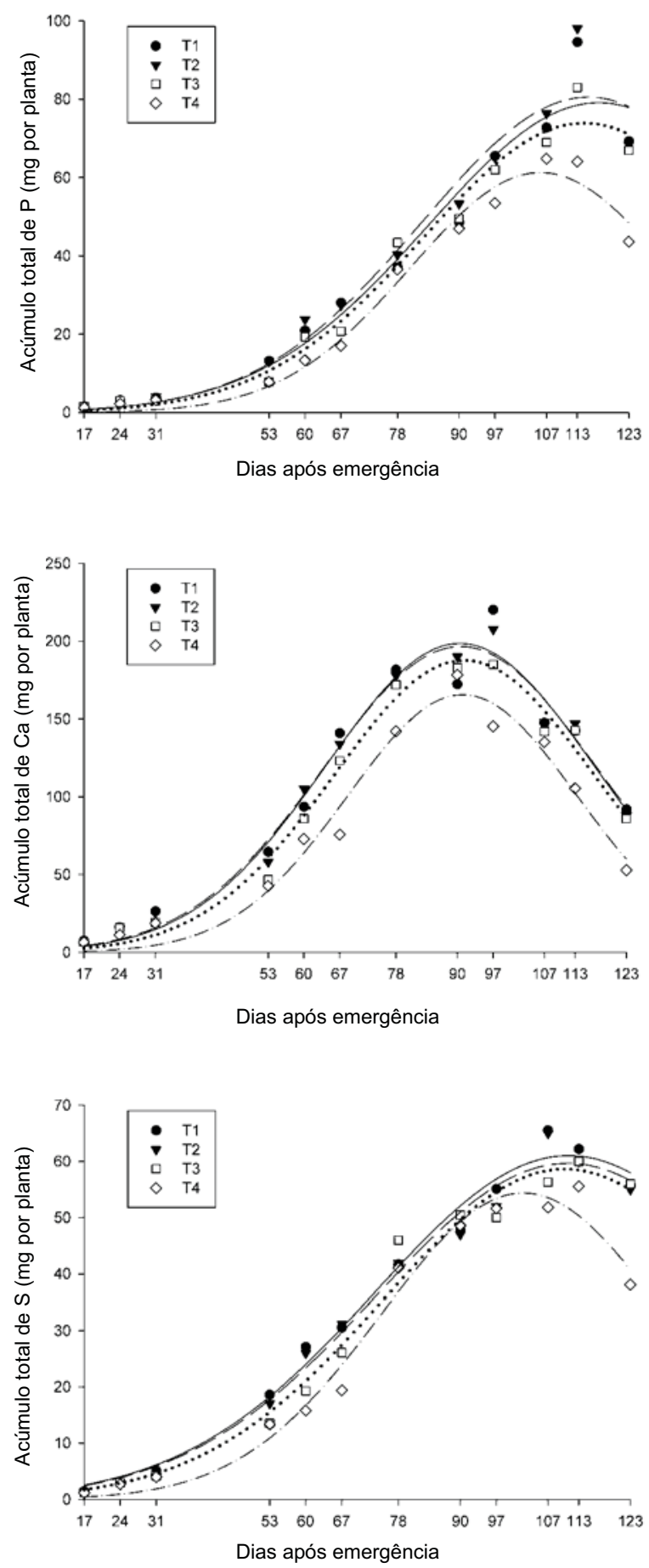

Figura 3 - Acúmulo de macronutrientes em função do tempo e de diferentes tratamentos. T1 = BRS 184 + capina manual (linha sólida); T2 = BRS 184 + herbicida seletivo (linha pontilhada); T3 = BRS 243 RR + capina manual (linha tracejada); T4 = BRS 243 RR + glyphosate (linha ponto-tracejada). 
Diversos fatores podem influenciar a absorção dos nutrientes, entre eles a capacidade de exploração do sistema radicular, as condições edafoclimáticas, as propriedades dos solos, a disponibilidade de água e,
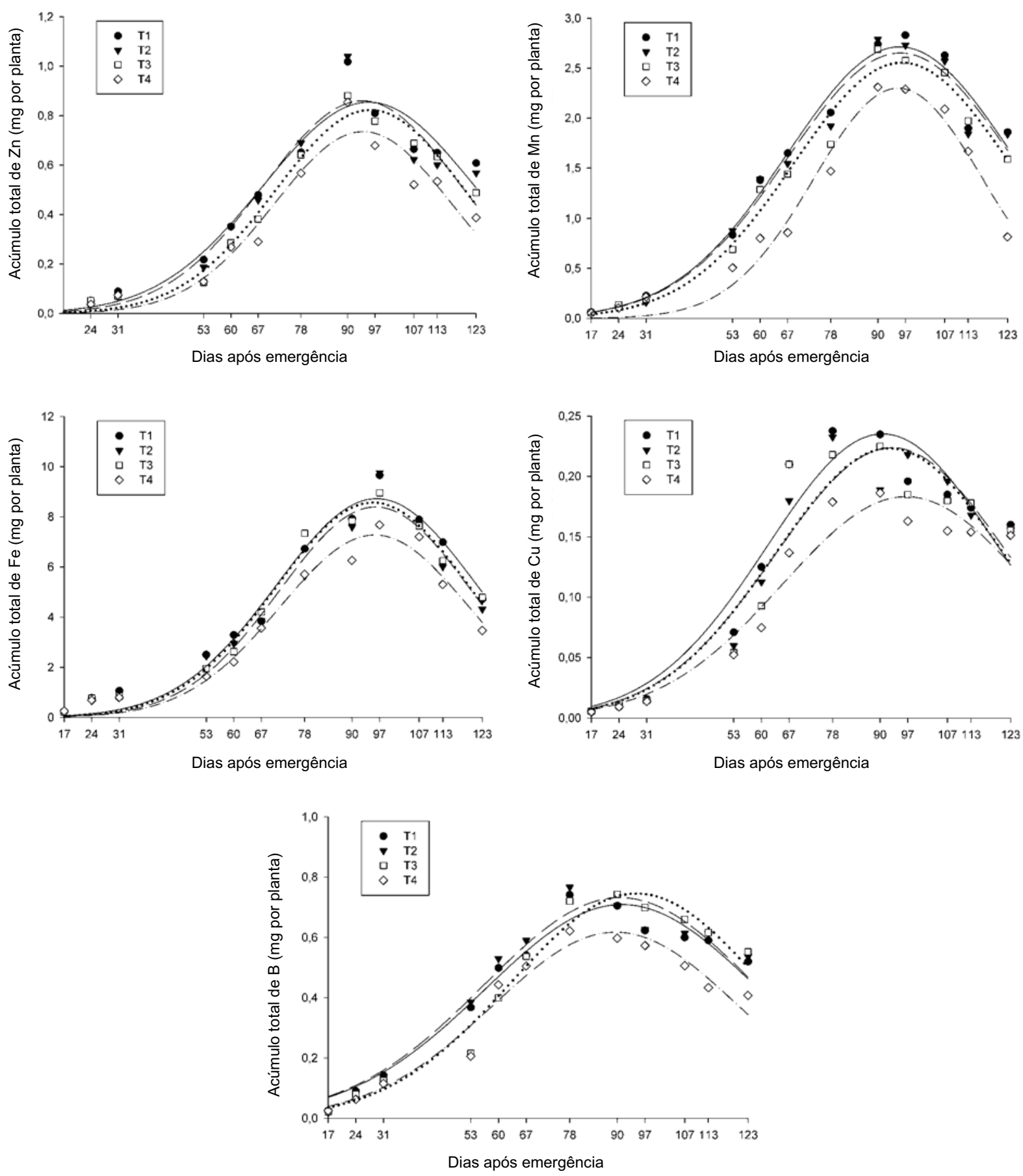

Figura 4 - Acúmulo de micronutrientes na soja em função do tempo e de diferentes tratamentos. T1 = BRS 184 + capina manual (linha sólida); T2 = BRS 184 + herbicida seletivo (linha pontilhada); T3 = BRS 243 RR + capina manual (linha tracejada); T4 = BRS 243 RR + glyphosate (linha ponto-tracejada). 
Tabela 4 - Estimativas dos parâmetros dos modelos ajustados para o acúmulo de macronutrientes secundários em função do tempo e os respectivos valores do ponto de inflexão (PI)

\begin{tabular}{|c|c|c|c|c|c|}
\hline \multirow{3}{*}{ Tratamento $^{1 /}$} & \multicolumn{3}{|c|}{ Estimativa dos parâmetros do modelo ajustado ${ }^{\underline{2} /}$} & \multirow{2}{*}{ PI } & \multirow{3}{*}{$\mathrm{R}^{2}$} \\
\hline & $\mathrm{a}$ & $\mathrm{X}_{0}$ & $\mathrm{~b}$ & & \\
\hline & (mg por planta) & \multicolumn{3}{|c|}{ (dias após a emergência) } & \\
\hline \multicolumn{6}{|c|}{ Cálcio } \\
\hline $\mathrm{T} 1$ & 196,61 & 90,57 & 26,56 & 64 & $0,98^{*}$ \\
\hline $\mathrm{T} 2$ & 198,62 & 90,48 & 26,12 & 64 & $0,99^{*}$ \\
\hline T3 & 187,71 & 91,32 & 25,45 & 65 & $0,99 *$ \\
\hline $\mathrm{T} 4$ & 165,62 & 90,97 & 22,46 & 68 & $0,98^{*}$ \\
\hline \multicolumn{6}{|c|}{ Magnésio } \\
\hline $\mathrm{T} 1$ & 84,23 & 102,37 & 35,96 & 66 & $0,97^{*}$ \\
\hline $\mathrm{T} 2$ & 86,94 & 99,79 & 33,32 & 66 & $0,98^{*}$ \\
\hline T3 & 82,35 & 99,58 & 32,50 & 67 & $0,98^{*}$ \\
\hline $\mathrm{T} 4$ & 67,43 & 98,86 & 31,19 & 67 & $0,98^{*}$ \\
\hline \multicolumn{6}{|c|}{ Enxofre } \\
\hline T1 & 61,04 & 111,02 & 37,37 & 73 & $0,99 *$ \\
\hline $\mathrm{T} 2$ & 59,67 & 110,83 & 37,14 & 73 & $0,99^{*}$ \\
\hline $\mathrm{T} 3$ & 58,63 & 110,31 & 35,17 & 75 & $0,99 *$ \\
\hline $\mathrm{T} 4$ & 54,37 & 102,18 & 27,46 & 74 & $0,99 *$ \\
\hline
\end{tabular}

1/ Somatório da MS das folhas, dos pecíolos, do caule, das vagens e dos grãos. ${ }^{2} \mathrm{a}=$ corresponde ao valor de máximo acúmulo; $\mathrm{x}_{0}=$ corresponde ao valor de $\mathrm{x}$, em DAE, que proporciona o máximo em $\quad$; b = corresponde à amplitude no valor de $\mathrm{x}$, em DAE, entre o ponto de inflexão e o ponto de máximo; T1 = BRS 184 + capina manual; T2 = BRS 184 + herbicida seletivo; T3 = BRS 243 RR + capina manual; $\mathrm{T} 4=$ BRS $243 \mathrm{RR}+$ glyphosate. $*(\mathrm{n}=5, \mathrm{P}<0.01)$.

soja transgênica, na presença do herbicida glyphosate (T4), resultaram em menor acúmulo de nutrientes em comparação aos demais tratamentos. Da mesma forma, comparando-se o máximo acúmulo de nutrientes obtido pelo parâmetro do modelo ajustado nas Tabelas 3, 4 e 5, o T4 apresentou sempre valores inferiores aos dos demais tratamentos.

Assim como na produção de matéria seca, observou-se que até os $31 \mathrm{DAE}$ o acúmulo de macro e micronutrientes foi baixo (Figuras 3 e 4). Entretanto, a partir dos $31 \mathrm{DAE}$, a taxa de acúmulo aumenta de forma acentuada, atingindo o ponto de máximo para os macronutrientes aos $104 \mathrm{DAE}$ e, para os micronutrientes, aos $94 \mathrm{DAE}$, havendo, no entanto, diferenças entre os tratamentos.

Como o valor do ponto de inflexão da curva corresponde ao dia após a emergência e o local onde o acúmulo dos macronutrientes da planta chegou ao valor máximo estimado, sendo a taxa de absorção diária crescente até o estádio fenológico, e levando em consideração a média dos valores de pontos de inflexão observados para a soja, tem-se que a taxa máxima de acúmulo de MS e nutrientes acontece aos 79, 73 e 66 DAE, respectivamente - valores próximos aos de Pedrinho Junior et al. (2004), que encontraram valores médios de máximo acúmulo de MS e macronutrientes aos 78 e 75 DAE, respectivamente. Esses autores também observaram acúmulo máximo de $\mathrm{N}, \mathrm{P}, \mathrm{K}$, $\mathrm{Ca}, \mathrm{Mg}$ e $\mathrm{S}$ aos 100, 108, 109, 111 e $106 \mathrm{DAE}$, respectivamente. Nesses estádios, os conteúdos de nutrientes na matéria seca foram de: $780 \mathrm{mg}$ por planta de N, $102 \mathrm{mg}$ por planta de $\mathrm{P}, 880 \mathrm{mg}$ por planta de $\mathrm{K}, 850 \mathrm{mg}$ por planta de $\mathrm{Ca}, 260 \mathrm{mg}$ por planta de $\mathrm{Mg}$ e $65 \mathrm{mg}$ por planta de $\mathrm{S}$. Exceto o $\mathrm{N}$ e $\mathrm{S}$, os valores de máximo acúmulo estimados para $\mathrm{P}, \mathrm{K}, \mathrm{Ca} \mathrm{e}$ $\mathrm{Mg}$ foram inferiores aos apresentados por Pedrinho Junior et al. (2004).

Os valores de máximo acúmulo para macro e micronutrientes não apresentaram diferenças estatísticas entre T1, T2 e T3. Resultados semelhantes foram obtidos por Zobiole et al. (2010d), os quais, ao avaliarem a influência de doses de glyphosate no acúmulo de nutrientes na soja RR, constataram que, com 
Tabela 5 - Estimativas dos parâmetros dos modelos ajustados para o acúmulo de micronutrientes em função do tempo e os respectivos valores do ponto de inflexão (PI)

\begin{tabular}{|c|c|c|c|c|c|}
\hline \multirow{3}{*}{ Tratamento $^{\underline{1} /}$} & \multicolumn{3}{|c|}{ Estimativa dos parâmetros do modelo ajustado ${ }^{2}$} & \multirow{2}{*}{ PI } & \multirow{3}{*}{$\mathrm{R}^{2}$} \\
\hline & \multirow{2}{*}{$\frac{\mathrm{a}}{\text { (mg por planta) }}$} & \multirow{2}{*}{$\mathrm{X}_{0}$} & \multirow{2}{*}{$\frac{\mathrm{b}}{\text { mergênc }}$} & & \\
\hline & & & & (dias após a emergência) & \\
\hline \multicolumn{6}{|c|}{ Zinco } \\
\hline $\mathrm{T} 1$ & 0,85 & 95,40 & 27,06 & 68 & $0,97^{*}$ \\
\hline $\mathrm{T} 2$ & 0,86 & 93,76 & 25,18 & 68 & $0,97^{*}$ \\
\hline T3 & 0,82 & 95,80 & 24,30 & 71 & $0,98^{*}$ \\
\hline $\mathrm{T} 4$ & 0,73 & 93,96 & 22,67 & 71 & $0,97^{*}$ \\
\hline \multicolumn{6}{|c|}{ Manganês } \\
\hline $\mathrm{T} 1$ & 2,71 & 95,51 & 28,54 & 66 & $0,98^{*}$ \\
\hline $\mathrm{T} 2$ & 2,65 & 95,66 & 28,53 & 67 & $0,98^{*}$ \\
\hline T3 & 2,55 & 96,10 & 27,47 & 68 & $0,99 *$ \\
\hline $\mathrm{T} 4$ & 2,29 & 95,16 & 21,51 & 73 & $0,98^{*}$ \\
\hline \multicolumn{6}{|c|}{ Ferro } \\
\hline $\mathrm{T} 1$ & 8,72 & 96,20 & 25,36 & 70 & $0,99 *$ \\
\hline $\mathrm{T} 2$ & 8,39 & 95,91 & 24,19 & 71 & $0,98^{*}$ \\
\hline T3 & 8,57 & 95,28 & 24,57 & 70 & $0,99^{*}$ \\
\hline T4 & 7,27 & 95,69 & 23,95 & 71 & $0,98^{*}$ \\
\hline \multicolumn{6}{|c|}{ Cobre } \\
\hline $\mathrm{T} 1$ & 0,23 & 90,61 & 29,07 & 61 & $0,97^{*}$ \\
\hline $\mathrm{T} 2$ & 0,22 & 92,96 & 29,41 & 63 & $0,97^{*}$ \\
\hline T3 & 0,22 & 92,40 & 29,02 & 63 & $0,96^{*}$ \\
\hline $\mathrm{T} 4$ & 0,18 & 96,50 & 31,06 & 65 & $0,97^{*}$ \\
\hline \multicolumn{6}{|c|}{ Boro } \\
\hline T1 & 0,71 & 91,18 & 34,41 & 56 & $0,98^{*}$ \\
\hline $\mathrm{T} 2$ & 0,73 & 90,64 & 34,20 & 56 & $0,98^{*}$ \\
\hline T3 & 0,74 & 94,84 & 31,56 & 63 & $0,98^{*}$ \\
\hline T4 & 0,61 & 89,52 & 30,95 & 58 & $0,97^{*}$ \\
\hline
\end{tabular}

1’ Somatório da MS das folhas, dos pecíolos, do caule, das vagens e dos grãos. ํㅚ a = corresponde ao valor de máximo acúmulo; $\mathrm{x}_{0}=$ corresponde ao valor de $\mathrm{x}$, em DAE, que proporciona o máximo em $; \mathrm{b}=$ corresponde à amplitude no valor de $\mathrm{x}$, em DAE, entre o ponto de inflexão e o ponto de máximo; T1 = BRS 184 + capina manual; T2 = BRS 184 + herbicida seletivo; T3 = BRS 243 RR + capina manual; $\mathrm{T} 4$ = BRS $243 \mathrm{RR}+$ glyphosate. * $(\mathrm{n}=5, \mathrm{P}<0,01)$.

o aumento da dose, houve diminuição no acúmulo de macro e micronutrientes. Essa diminuição, provavelmente, deveu-se à menor taxa fotossintética da planta e menor absorção de nutrientes (Eker et al., 2006; Bott et al., 2008).

A exigência nutricional variou com a fase do ciclo de desenvolvimento da soja, tanto para macro quanto para micronutrientes (Figuras 3 e 4), e acompanha a taxa de acúmulo de matéria seca durante o estádio vegetativo até o final do florescimento (Figura 1).
Entretanto, o máximo acúmulo diário dos nutrientes coincidiu com o período inicial de frutificação de ambos os tratamentos, quando ocorre o estabelecimento de uma força mobilizadora de nutrientes e assimilados, devido ao aumento da atividade metabólica, associada à atividade hormonal e à divisão e crescimento celular (Taiz \& Zeiger, 1995). Considerando a média dos pontos de máximo acúmulo dos nutrientes, a ordem de extração para os macronutrientes foi: $\mathrm{N}>\mathrm{K}>\mathrm{Ca}>\mathrm{Mg}$ $>\mathrm{P}>\mathrm{S}$, e para os micronutrientes: $\mathrm{Fe}>\mathrm{Mn}>$ $\mathrm{Zn}>\mathrm{B}>\mathrm{Cu}$. 
Tabela 6 - Peso de 100 grãos (g) de plantas de soja convencional e transgênica, submetida a diferentes tratamentos de controle de plantas daninhas

\begin{tabular}{|c|c|c|c|}
\hline \multicolumn{4}{|c|}{ Tratamento (g por planta) } \\
\hline $\mathrm{T} 1$ & $\mathrm{~T} 2$ & $\mathrm{~T} 3$ & $\mathrm{~T} 4$ \\
\hline $15,18^{*} \mathrm{a}$ & $15,64 \mathrm{a}$ & $10,80 \mathrm{~b}$ & $11,05 \mathrm{~b}$ \\
\hline $\mathrm{DMS}=0,49$ & \multicolumn{3}{|c|}{$\mathrm{CV} \%=2,42$} \\
\hline \multicolumn{4}{|c|}{$\mathrm{D} \%$} \\
\hline
\end{tabular}

* Médias dentro das colunas seguidas pelas mesmas letras não são significativas a 5\% de probabilidade pelo teste LSD. T1 = BRS 184 + capina manual; T2 = BRS $184+$ herbicida seletivo; T3 = BRS 243 RR + capina manual; T4 = BRS 243 RR + glyphosate.

Segundo Sfredo \& Sarruge (1990), a dinâmica da absorção de nutrientes pelas plantas é um fator que influencia no desempenho de uma cultura em determinada região ou país. Nesse sentido, o menor acúmulo de nutrientes no T4 levou ao menor peso de 100 sementes (Tabela 5); no entanto, em relação à produtividade, não houve diferença significativa entre os tratamentos (Tabela 6).

Os resultados obtidos são semelhantes aos de Zobiole et al. (2010a), os quais sugeriram que cultivares convencionais de soja podem apresentar niveis críticos de nutrientes diferentes comparados com os da soja RR tratada com glyphosate, pois o nível crítico de determinado nutriente na planta pode ser variável em função das variações na capacidade de absorver e/ou utilizar o nutriente (Fageria, 1976; Muniz et al., 1985; Fageria, 1987; Fonseca et al., 1988, Scherer, 1998). A menor produção de biomassa nos cultivares RR tratados com glyphosate indica que um nivel maior de nutrientes pode ser requerido por esses cultivares para atingir a eficiência fisiológica e que eventualmente novos parâmetros deverão ser estabelecidos para a otimização da nutrição mineral da soja RR tratada com glyphosate, tendo em vista os efeitos desse herbicida no metabolismo da planta.

\section{AGRADECIMENTOS}

Ao Conselho Nacional de Pesquisa e Desenvolvimento (CNPq), pela bolsa de estudos e pelo apoio financeiro, e à Embrapa Soja, pelo apoio para a realização da pesquisa e condução do experimento. As marcas comerciais no texto são apenas informativas e não representam nenhuma recomendação oficial da Universidade Estadual de Maringá, Embrapa e USDA-ARS.

\section{LITERATURA CITADA}

BOTT, S. et al. Glyphosate-induced impairment of plant growth and micronutrient status in glyphosate-resistant soybean (Glycine max L.). Plant Soil, v. 312, n. 1, p. 185-194, 2008.

CAKMAK, I. et al. Glyphosate reduced seed and leaf concentrations of calcium manganese, magnesium and iron in non-glyphosate resistant soybean. Eur. J. Agron., v. 31, n. 1, p. 114-119, 2009.

COUTINHO, C. F. B.; MAZO, L. H. Metallic complex with glyphosate: a review. Química Nova, v. 28, n. 6, p. 1038-1045, 2005.

DVORANEN, E. C. et al. Nodulação e crescimento de variedades de soja rr sob aplicação de glyphosate, fluazifopp-butyl e fomesafen. Planta Daninha, v. 26, n. 3, p. 619-625, 2008.

EKER, S. et al. Foliar-applied glyphosate substantially reduced uptake and transport of iron and manganese in sunflower (Helianthus annuus L.) plants. J. Agric. Food Chem., v. 54, n. 27, p. 10019-10025, 2006.

FAGERIA, N. K. Critical P, K, Ca and Mg contents in the tops of rice and peanut plants. Plant Soil, v. 45, n. 2, p. 421-431, 1976.

FAGERIA, N. K. Variação em diferentes estádios de crescimento do nível critico de fósforo em plantas de arroz. R. Bras. Ci. Solo, v. 11, n. 1, p. 77-80, 1987.

FONSECA, D. M. et al. Níveis críticos de fósforo em amostras de solos para o estabelecimento de Andropogon gayanus, Brachiaria decumbens e Hyparrhenia rufa.

R. Bras. Ci. Solo, v. 12, n. 1, p. 49-58, 1988.

EMPRESA BRASILEIRA DE PESQUISA AGROPECUÁRIA - EMBRAPA. Manual de análises químicas de solos, plantas e fertilizantes. Brasília: 1999. $370 \mathrm{p}$.

EMPRESA BRASILEIRA DE PESQUISA AGROPECUÁRIA - EMBRAPA. Sistema brasileiro de classificação de solos. 2.ed. Rio de Janeiro: Embrapa Solos, 2006. 306 p.

EMPRESA BRASILEIRA DE PESQUISA AGROPECUÁRIA - EMBRAPA. Tecnologias de produção de soja - Paraná. Londrina: Embrapa/CNPSo, 2007. 218 p. 
FRANZEN, D. W.; O’BARR, J. H.; ZOLLINGER, R. K. Interaction of a foliar application of iron HEDTA and three postemergence broadleaf herbicides with soybeans stressed from chlorosis. J. Plant Nutr., v. 26, n. 12, p. 2365-2374, 2003.

JAWORSKI, E. G. Mode of action of N-phosphonomethylglycine: inhibition of aromatic amino acid biosynthesis. J. Agric. Food Chem., v. 20, n. 6, p. 1195-1198, 1972.

JOHAL, G. S.; HUBER, D. M. Glyphosate effects on disease and disease resistance in plants. Eur. J. Agron., v. 31, n. 3, p. 144-152, 2009.

KING, A. C.; PURCELL, L. C.; VORIES, E. D. Plant growth and nitrogenase activity of glyphosate-tolerant soybean in response to glyphosate applications. Agron. J., v. 93, n. 1, p. 179-186, 2001.

KITCHEN, L. M.; WITT, W. W.; RIECK, C. E. Inhibition of chlorophyll accumulation by glyphosate. Weed Sci., v. 29, n. 4, p. 513-516, 1981.

MUNIZ, A. S. et al. Nível crítico de fósforo na parte aérea da soja como variável do fator capacidade de fósforo no solo.

R. Bras. Ci. Solo, v. 9, n. 3, p. 237-243, 1985.

OLIVEIRA JR., R. S. et al. Influência do glyphosate sobre a nodulação e o crescimento de vinte cultivares de soja RR.

Planta Daninha, v. 26, n. 4, p. 831-843, 2008.

PEDRINHO JUNIOR, A. F. F.; BIANCO, S.; PITELLI, R. A. Acúmulo de massa seca e macronutrientes por plantas de Glycine max e Richardia brasiliensis. Planta Daninha, v. 22, n. 1, p. 53-61, 2004.

REDDY, K. N.; HOAGLAND, R. E.; ZABLOTOWICZ, R. M. Effect of glyphosate on growth, chlorophyll content and nodulation in glyphosate-resistant soybeans (Glycine max) varieties. J. New Seeds., v. 2, n. 1, p. 37-52, 2000.
REDDY, K. N.; RIMANDO, A. M.; DUKE, S. O. Aminomethylphosphonic acid, a metabolite of glyphosate, causes injury in glyphosate-treated, glyphosate-resistant soybean. J. Agric. Food Chem., v. 52, n. 16, p. 5139-5143, 2004.

SARRUGE, J. R., HAAG, H. P. Análise química em plantas. Piracicaba: ESALQ, 1974. 56 p.

SCHERER, E. E. Níveis críticos de potássio para a soja em Latossolo húmico de Santa Catarina. R. Bras. Ci. Solo, v. 22, n. 1, p. 57-62, 1998.

SFREDO, G. J.; SARRUGE, J. R. Acúmulo de micronutrientes em plantas de girassol. Pesq. Agropec. Bras., v. 25, n. 4, p. 499-503, 1990.

TAIZ, L.; ZEIGER, E. Plant physiology. Sunderland: Sinauer Associates, 1998. 719 p.

ZABLOTOWICZ, R. M.; REDDY, K. N. Nitrogenase activity, nitrogen content, and yield responses to glyphosate in glyphosate-resistant soybean. Crop Protec., v. 26, n. 3, p. 370-376, 2007.

ZOBIOLE, L. H. S. et al. Glyphosate reduces shoot concentration of mineral nutrients in glyphosateresistant soybeans. Plant Soil, v. 328, n. 1, p. 57-69, 2010a.

ZOBIOLE, L. H. S. et al. Uso de aminoácido exógeno na prevenção de injúrias causadas por glyphosate na soja RR. Planta Daninha, v. 28, n. 3, p. 643-653, 2010b.

ZOBIOLE, L. H. S. et al. Water use efficiency and photosynthesis of glyphosate-resistant soybean as affected by glyphosate. Pest. Biochem. Physiol., v. 97, n. 1, p. 182-193, 2010c.

ZOBIOLE, L. H. S. et al. Nutrient accumulation and photosynthesis in glyphosate resistant soybeans is reduced under glyphosate use. J. Plant Nutr., v. 33, n. 1, p. 1860-1873, 2010d. 